\title{
Presence of large scavenger receptor A-positive cells in systemic circulation: A potential prognostic indicator for idiopathic pulmonary fibrosis, connective tissue disease- associated interstitial lung disease, acute exacerbation and risk factor for acute exacerbation
}

\author{
Iwao Emura* and Hiroyuki Usuda \\ Department of Surgical Pathology, Japanese Red Cross Nagaoka Hospital, Nagaoka city, Japan
}

\begin{abstract}
Background: Acute Exacerbation (AE) of Idiopathic Pulmonary Fibrosis (IPF) and Connective Tissue Disease (CTD)-associated Inflammatory Lung Disease (ILD) are considered systemic disorders that may lead to multiple organ injury. Presumably, large scavenger receptor A-positive (SRA $)$ cells in systemic circulation play a central role in multiple organ injury. We investigated the utility of large $\mathrm{SRA}^{+}$cells as a risk factor for $\mathrm{AE}$ and prognostic factor for IPF, CTD-associated ILD, and AE.

Methods: A total of 159 subjects with IPF or CTD-associated ILD and 71 with AE were examined. Fresh peripheral blood samples were examined with immune cytochemical method. Patients with IPF or CTD-associated ILD were classified into group A (large SRA ${ }^{+}$cells were not detected before the onset of an AE), group $\mathrm{B}$ (large $\mathrm{SRA}^{+}$cells were detected before the onset of an $\mathrm{AE}$ ), and patients with $\mathrm{AE}$ were classified into group $\mathrm{C}$ (large $\mathrm{SRA}^{+}$cells were not detected at the onset or after the onset of an $\mathrm{AE}$ ) and group $\mathrm{D}$ (large $\mathrm{SRA}^{+}$cells were detected at the onset or after the onset of an $\mathrm{AE}$ ), and were followed up for 1 year.

Findings: Sixteen of 114 Group A patients and 23 of 45 of Group B developed AE. One of 15 Group C patients and 47 of 56 Group D died. The AE rate and the mortality rate were significantly higher in Group B and D than Group A and C. Kaplan-Meier curves for AE and survival of Group B and D were significantly worse than those of Group A and C.
\end{abstract}

Interpretation: Our data indicated that the appearance of large SRA+ cells in systemic circulation might be a useful risk factor for AE, and prognostic factor for IPF, CTD-associated ILD, and AE.

\section{Introduction}

Many patients with Idiopathic Pulmonary Fibrosis (IPF) may experience sudden worsening of respiratory symptoms [1]. This frequently occurs without an identifiable cause, and is termed Acute Exacerbation (AE) of IPF [2]. Currently, AE have now also been reported in patients with Connective Tissue Disease (CTD)-associated interstitial lung disease (ILD) and other ILDs [3,4]. Respiratory failure from AE is associated with high in-hospital mortality [5-7]. For this reason, sensitive biomarkers and prognostic factors are necessary to predict the onset of $\mathrm{AE}$ as well as the need for early interventions. Several physiologic candidate risk factors and prognostic factors have been reported [8]. Elevated serum level of Krebs von den Lungen-6 (KL-6) at baseline has been associated with increased risk for AE-IPF [9], but results were conflicting [10].

In a previous report, we reported that 1) AE of IPF and CTDassociated ILD as systemic disorders that lead to multiple organ injury, 2) large scavenger receptor $A$ positive $\left(S_{R A}{ }^{+}\right)$cells, tumor necrosis factor (TNF)- $\alpha$ positive cells and neutrophils in systemic circulation played a central role in multiple organ injury, and 3) large $\mathrm{SRA}^{+}$ cells had been detected in many patients before the onset of an $\mathrm{AE}$, conversely, TNF- $\alpha^{+}$cells had been detected after the onset of an AE [11].
The aim of this study was to investigate whether the presence of large $\mathrm{SRA}^{+}$cells in systemic circulation is as a risk factor for $\mathrm{AE}$ and whether it can serve as a prognostic factor for IPF, CTD-associated ILD, and AE thereof.

\section{Methods}

Patients with IPF and CTD-associated ILD (during April 2009 and October 2015) and those with AE (during April 2005 and October 2015) were examined. The Japanese Red Cross Nagaoka Hospital ethical committee approved the study (No. 406).

\section{IPF and CTD-associated ILD group}

A total of 159 consecutive patients with IPF or CTD-associated ILD were chosen for the present study (Table 1). All patients were classified

Correspondence to: Iwao Emura, Department of Surgical Pathology, Japanese Red Cross Nagaoka Hospital, 2-297-1 Senshyuu, Nagaoka City, Niigata Prefecture, Japan, Tel: 0258-28-3600, Fax: 0258-28-9000, E-mail. emura@nagaoka.jrc.or.jp

Received: September 02, 2017; Accepted: September 28, 2017; Published: September 30, 2017 
Emura I (2017) Presence of large scavenger receptor A-positive cells in systemic circulation: A potential prognostic indicator for idiopathic pulmonary fibrosis, connective tissue disease-associated interstitial lung disease, acute exacerbation and risk factor for acute exacerbation

Table 1. Baseline characteristics of the study population with idiopathic pulmonary fibrosis and collagen disease associated-inflammatory lung disease, and acute exacerbation rate.

\begin{tabular}{|c|c|c|c|}
\hline Variable & Group A (n=114) & Group B (n=45) & $\boldsymbol{p}$ \\
\hline Age & $65.2 \pm 9.9$ & $67.7 \pm 9.6$ & 0.139 \\
\hline Sex (M) n, (\%) & $57(50 \%)$ & $21(47 \%)$ & 0.42 \\
\hline KL-6 & $1663 \pm 1388$ & $1241 \pm 585$ & 0.008 \\
\hline Coll. Dis. n, (\%) & $43(37 \%)$ & $16(35 \%)$ & 0.474 \\
\hline Steroid n, (\%) & $82(72 \%)$ & $24(53 \%)$ & 0.04 \\
\hline Pulse n, (\%) & $9(8 \%)$ & $8(18 \%)$ & 0.088 \\
\hline Pulse + CMP n, (\%) & $9(8 \%)$ & $12(26 \%)$ & 0.003 \\
\hline AE n, (\%) & $16(14 \%)$ & $23(51 \%)$ & $<0.001$ \\
\hline
\end{tabular}

n: number, Coll. Dis.: Collagen disease, Pulse: Steroid pulse therapy, CPM: cyclophosphamide, AE: Acute exacerbation

into two groups: Group A patients (114 cases) without large $\mathrm{SRA}^{+}$cells in cytological preparations before the onset of an AE, and Group B (45 cases) with one or more large $\mathrm{SRA}^{+}$cells in cytological preparations identified at one or more-time points before the onset of an AE. Patients in Group A were followed for 1 year from hospitalization. Large SRA ${ }^{+}$cells were detected during the clinical course in many of group B patients. So, patients in Group B were followed for 1 year from the detection of large $\mathrm{SRA}^{+}$cells to assess the utility of the presence of large $\mathrm{SRA}^{+}$cells as a risk factor for AE.

\section{Acute exacerbation group}

A total of 71 consecutive patients with AE of IPF or CTDassociated ILD were chosen for the present study (Table 2). All patients were classified into Group C (large SRA ${ }^{+}$cells were not detected in cytological preparations at the onset or after the onset of an AE: 15 cases) or Group D (large SRA ${ }^{+}$cells were detected at the onset or after the onset of an AE: 56 cases), and were followed for 1 year from the onset of an AE.

Patients whose immediate causes of death were considered to be severe bronchopneumonia, aspiration pneumonia, lung cancer, heart failure or bacterial sepsis were excluded from the analysis.

\section{Cytology}

Blood samples of 159 Group A and B patients obtained before the onset, at the onset and after the onset of an AE were examined, but those of 32 Group C and D patients obtained before the onset of an AE (14 patients who developed AE during 2005 to 2008 and 18 patients whose diagnosis at admission was AE) were not examined. The rest of fresh peripheral blood samples obtained for the examination of differential white blood counts during the hospitalization were examined (median times [range], 4 [1-18]). Peripheral blood was collected into tubes containing EDTA (Vacutainer plastic, EDTA 2K; Becton Dickinson, Franklin Lakes, NJ, USA). Red blood cells were lysed with lysing

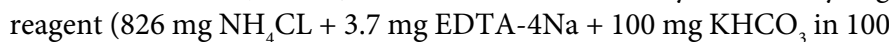
$\mathrm{ml} \mathrm{H}_{2} \mathrm{O}$ ). Nucleated cells were suspended in isotonic sodium chloride solution. Suspensions containing about $5 \times 10^{6}$ nucleated cells were smeared on glass slides using Auto smear CF-12 (Sakura Seiki, Tokyo, Japan). Cells that did not adhere to glass slides were gently washed off with $95 \%$ ethanol solution. Approximately $1.0 \times 10^{6}$ nucleated cells were evenly smeared in one $1.2 \times 1.2 \mathrm{~cm}^{2}$ area. Smear preparations were fixed in $95 \%$ ethanol solution and stained using the Papanicolaou method.

\section{Immunocytochemistry}

Papanicolaou-stained smears were used for examination, which was performed with the simple stain MAX-PO method (NICHIREI Co., Tokyo, Japan) and with diaminobenzidine as the chromogen using mouse monoclonal anti-human SRA antibody (CD204, a macrophage SRA marker, 1:200; Trans Genic Inc., Kumamoto, Japan). An antigen retrieval method using citrate buffer and microwave heating was employed. As a negative control, the primary antibody was substituted by phosphate-buffered saline. In these controls, no positive stain was observed.

KL-6: We measured KL-6 levels (median times [range], 2 [1-5]) using the KL-6 electrochemiluminescence immunoassay kit (EIDIA Co., Ltd., Tokyo, Japan) with $<500 \mathrm{U} / \mathrm{ml}$ as the reference value. The highest level of KL- 6 during the hospitalization of each patient was presented.

Diagnoses: We chose the patients who met the diagnostic criteria of IPF or AE-IPF $[12,13]$. The diagnosis of AE of CTD-associated ILD was made using the same criteria as IPF. SRA ${ }^{+}$cells in peripheral blood that were over twice as large as monocytes were defined as large SRA ${ }^{+}$ cells, and SRA ${ }^{+}$cells smaller than large SRA ${ }^{+}$cells were defined as small $\mathrm{SRA}^{+}$cells.

\section{Statistical analysis}

All continuous variables were reported as mean $\pm \mathrm{SD}$. Continuous variables were compared by a t-test or Fisher's exact test. Discrete variables were analyzed by using the chi-square test. Survival analyses were performed by using the Kaplan-Meier method and log-rank tests. In all analyses, a $p$-value less than 0.05 was considered to be statistically significant. The software SPSS statistics version 17.0 (SPSS Japan Inc., Tokyo, Japan) was used for data management and statistical analyses.

\section{Results}

\section{Study population}

IPF and CTD-associated ILD group: Baseline characteristics are shown in Table 1. Ninety-three of 152 cases satisfied the diagnostic criteria for IPF and 59 patients presented CTD-associated ILD. Percent vital capacity were measured 1 or 2 times during admission in 81 patients (Group A: 54, B:27). We chose lower value, and there was no significant difference between group A $(68.0 \pm 13.2)$ and $B(65.1 \pm 15.9)$.

Acute exacerbation group: Of 71 cases, 14 patients developed $\mathrm{AE}$ during 2005 to 2008. Thirty-nine patients were from the Group A and B. Nine of 16 cases from Group A were classified into Group C, and 7 of the 16 cases and all 23 from Group B were classified into Group D. The diagnosis at admission was AE in 18 cases. Baseline characteristics are shown in Table 2.

\section{Cytological findings}

Small SRA ${ }^{+}$cells were detected in all cases. Only one to five large $\mathrm{SRA}^{+}$cells were observed in some of the smears of patients in Group B

Table 2. Baseline characteristics of the study population with acute exacerbation and the mortality rate.

\begin{tabular}{|c|c|c|c|}
\hline Variable & Group A (n=15) & Group B $(n=56)$ & $p$ \\
\hline Age & $70.5 \pm 8.5$ & $69.4 \pm 10.0$ & 0.679 \\
\hline $\operatorname{Sex}(M) n,(\%)$ & $6(40 \%)$ & $30(54 \%)$ & 0.397 \\
\hline KL-6 & $2657 \pm 2245$ & $1633 \pm 1004$ & 0.105 \\
\hline Coll. Dis. n, (\%) & $3(20 \%)$ & $13(23 \%)$ & 1 \\
\hline Steroid n, (\%) & $5(33 \%)$ & $7(13 \%)$ & 0.113 \\
\hline Pulse $n,(\%)$ & $4(27 \%)$ & $18(32 \%)$ & 0.763 \\
\hline Pulse + CPM n, (\%) & $6(40 \%)$ & $31(55 \%)$ & 0.386 \\
\hline Death $n,(\%)$ & $1(7 \%)$ & $47(84 \%)$ & $<0.001$ \\
\hline
\end{tabular}


Emura I (2017) Presence of large scavenger receptor A-positive cells in systemic circulation: A potential prognostic indicator for idiopathic pulmonary fibrosis, connective tissue disease-associated interstitial lung disease, acute exacerbation and risk factor for acute exacerbation

before the onset of an $\mathrm{AE}$ (Figure 1). Large SRA ${ }^{+}$cells were detected before the onset or at the onset of an $\mathrm{AE}$ in 49 of 56 Group D patients $(87.5 \%)$, and after the onset of an AE in 7 group D patients from Group A (12.5\%).

\section{Risk factor}

Sixteen of $114(14 \%)$ patients in Group A and 23 of $45(51 \%)$ in Group B developed AE. The AE rate was significantly higher in Group B than Group A (Table 1, $p<0.001$ ). The Kaplan-Meier curve of Group B showed a significantly worse AE than in Group A. (Figure 2, $p<0.001$ ).

\section{Mortality}

One of 15 (7\%) patients in Group C and 47 of 56 (84\%) in Group D died of respiratory failure. The mortality rate of was significantly higher

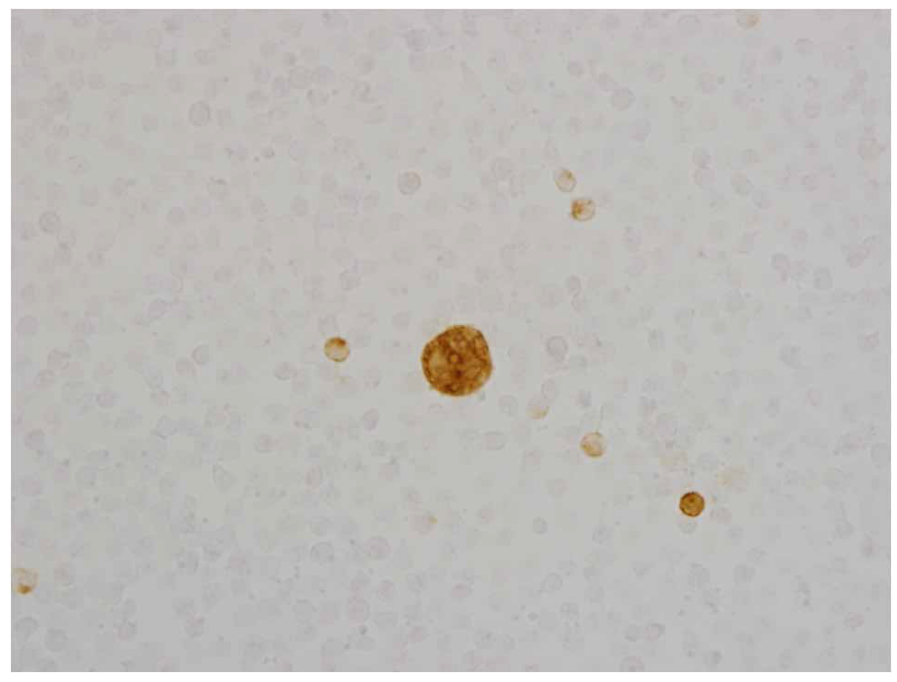

Figure 1. Scavenger receptor A positive cells in peripheral blood. Footnote: Large and small scavenger receptor A-positive cells.

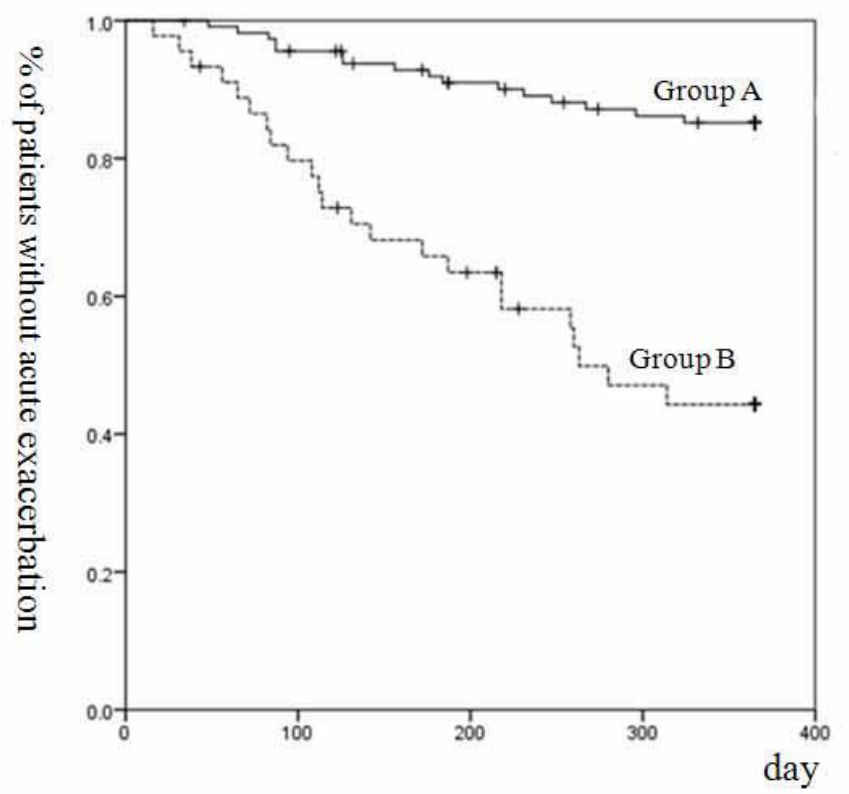

Figure 2. Kaplan-Meier analysis for the onset of an acute exacerbation of IPF and CTD associated ILD.

Footnote: Kaplan-Meier survival curve for acute exacerbation of group B was significantly worse than that of Group A. ( $p<0.001$, log-rank test). IPF, idiopathic pulmonary fibrosis; CTD, connective tissue disease; ILD, inflammatory lung disease

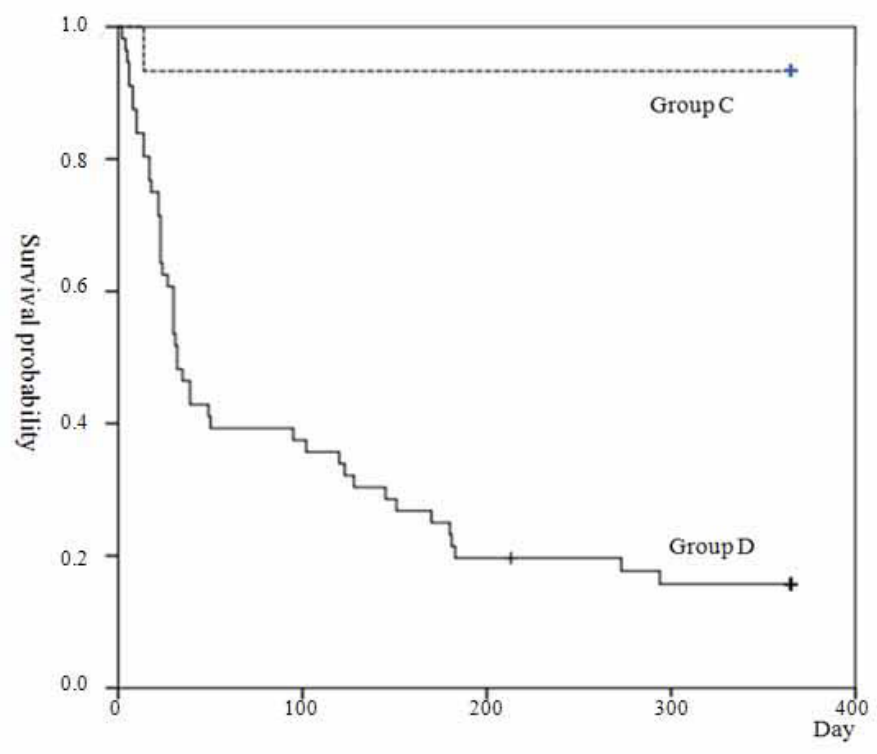

Figure 3. Kaplan-Meier survival curve for acute exacerbation groups.

Footnote: Kaplan-Meier survival curves of group D showed significantly worse survival than Group C ( $<<0.001$, log-rank test).

in Group D than in Group C (Table 2, $p<0.001)$. Kaplan-Meier survival curves of Group D showed significantly worse survival than in Group C (Figure 3, $p<0.001$ ).

\section{Discussion}

The present our data indicated that the appearance of large SRA ${ }^{+}$ cells in systemic circulation might be a useful risk factor for $\mathrm{AE}$, and prognostic factor for IPF, CTD-associated ILD, and AE.

Forty-eight of $71(67.6 \%)$ patients with AE (Group C: 1 and Group D: 47) died. Our present results support those of previous reports indicating that respiratory failure from AE of IPF or CTD-associated ILD is associated with high in-hospital mortality [5-7]. Furthermore, the AE rate was significantly higher in Group B than Group A, and the Kaplan-Meier curve of Group B showed a significantly worse AE than in Group A. All 23 patients in Group B who developed AE were classified into Group D. The mortality rate of was significantly higher in Group D than in Group C. Kaplan-Meier survival curves of Group D showed significantly worse survival than in Group C. From these data, the presence of large $\mathrm{SRA}^{+}$cells seemed to be a useful indicator of the poor prognosis of IPF, CTD-associated ILD, and AE.

The AE rate was significantly higher in Group B than in Group A. The Kaplan-Meier curve for AE of Group B was significantly worse than that of Group A. Our present data indicated that the appearance of large $\mathrm{SRA}^{+}$cells might be a useful risk factor for $\mathrm{AE}$.

Diffuse alveolar damage is frequently observed in patients who have died from an AE of IPF [14-16], but pathological findings of systemic organs were not described in these reports. Autopsy was performed in 14 of 47 Group D patients and we reported that 1) Multiple organ injury (including diffuse alveolar damage) was confirmed in all cases, 2) Large $\mathrm{SRA}^{+}$cells and TNF- $\alpha^{+}$cells in systemic circulation were considered to play central roles in multiple organ injury. 3) large SRA ${ }^{+}$ cells were detected before the onset of an AE and TNF- $\alpha^{+}$cells were detected after the onset of an $\mathrm{AE}$ in all cases [11]. In the present study, large $\mathrm{SRA}^{+}$cells were detected before the onset or at the onset of an $\mathrm{AE}$ in 45 Group $\mathrm{B}$ patients, and after the onset of an $\mathrm{AE}$ in 7 group $\mathrm{D}$ 
Emura I (2017) Presence of large scavenger receptor A-positive cells in systemic circulation: A potential prognostic indicator for idiopathic pulmonary fibrosis, connective tissue disease-associated interstitial lung disease, acute exacerbation and risk factor for acute exacerbation

patients from Group A. These finding indicate that during the clinical course, the disease continued to progress, even after the onset of an $\mathrm{AE}$, eventually leading to multiple organ injury.

Large $\mathrm{SRA}^{+}$cells were detected in multiple organ dysfunction syndrome cases (including ARDS cases) [16], and the coincidence of the appearance of large $\mathrm{SRA}^{+}$cells and increase of small SRA ${ }^{+}$cells might be useful to predict poor outcome in patients with systemic inflammatory response syndrome or multiple organ dysfunction syndrome [17]. We speculated that large $\mathrm{SRA}^{+}$cells differentiated from monocytes in systemic circulation. SRA-negative monocytes become positive for SRA by the stimulation of macrophage colonystimulating factor $[18,19]$. Endothelial cells, fibroblasts, etc. have an ability to produce one or other colony stimulating factors [20], and are activated locally by injury of cells and tissues to induce production and secretion of cytokines [21]. ARDS develops secondary to a wide range of primary insults [22]. We could find definite primary insults responsible for the differentiation of large $\mathrm{SRA}^{+}$cells in each our multiple organ dysfunction syndrome case [16]. However, we could not find any evidence of extra-pulmonary primary insults responsible for the differentiation of large SRA ${ }^{+}$cells in IPF and CTD-associated ILD cases [11]. It seemed important to find definite primary insult in IPF and CTD-associated ILD cases for the better understanding of AE.

We acknowledge certain limitations of this study. First, we consider that the cytological method used in this study for the examination of peripheral blood was originally developed by us. Thus, we could not find any papers discussing the presence of large $\mathrm{SRA}^{+}$cells in the peripheral blood of patients with IPF, CTD-associated ILD, or AE. We could not compare our results with those of other reports. Second, as this was a single-center study, the patient population and standards of care may vary from those in other centers. Thus, the reported results may not be broadly representative. However, the cytological method is used worldwide and the presence of large $\mathrm{SRA}^{+}$cells in systemic circulation may be an attractive marker, as blood is readily available, and its measurement can be easily standardized. Future studies are necessary to confirm the clinical utility of the presence of large SRA ${ }^{+}$ cells in circulation as a risk and prognostic factor for patients with IPF, CTD-associated ILD, or AE.

\section{Acknowledgement}

The authors sincerely express their appreciation to Dr. Shinichi Toyabe (Crisis Management Office, Niigata University) for his statistical analysis.

\section{References}

1. Kim DS, Collard HR, King TE Jr (2006) Classification and natural history of the idiopathic interstitial pneumonias. Proc Am Thorac Soc 3: 285-292. [Crossref]

2. Kondoh Y, Taniguchi H, Kawabata Y, Yokoi T, Suzuki K, et al. (1993) Acute exacerbation in idiopathic pulmonary fibrosis. Analysis of clinical and pathologic findings in three cases. Chest 103:1808-1812. [Crossref]

3. Park IN, Kim DS, Shim TS, Lim CM, Lee SD, et al. (2007) Acute exacerbation of interstitial pneumonia other than idiopathic pulmonary fibrosis. Chest 132: 214-220. [Crossref]

4. Churg A, Müller NL, Silva CI, Wright JL (2007) Acute exacerbation (acute lung injury of unknown cause) in UIP and other forms of fibrotic interstitial pneumonias. Am J Surg Pathol 31: 277-284. [Crossref]

5. Song JW, Hong SB, Lim CM, Koh Y, Kim DS (2011) Acute exacerbation of idiopathic pulmonary fibrosis: incidence, risk factors and outcome. Eur Respir J 37: 356-363. [Crossref]

6. Kishaba T, Tamaki H, Shimaoka Y, Fukuyama H, Yamashiro S (2014) Staging of acute exacerbation in patients with idiopathic pulmonary fibrosis. Lung 192: 141-149. [Crossref]
7. Akira M, Kozuka T, Yamamoto S, Sakatani M (2008) Computed tomography findings in acute exacerbation of idiopathic pulmonary fibrosis. Am J Respir Crit Care Med 178 372-378. [Crossref]

8. Collard HR, Rysrson CJ, Corte TJ, Jenkins G, Kondoh Y, et al. (2016) Acute exacerbation of idiopathic pulmonary fibrosis. An international working group report. Am J Respir Crit Care Med 194: 265-275. [Crossref]

9. Ohshimo S, Ishikawa N, Horimasu Y, Hattori N, Hirohashi N, et al. (2014) Baseline KL-6 predicts increased risk for acute exacerbation of idiopathic pulmonary fibrosis. Respir Med 108: 1031-1039. [Crossref]

10. Richeldi L, du Bois RM, Raghu G, Azuma A, Brown KK, et al. (2014) Efficacy and safety of nintedanib in idiopathic pulmonary fibrosis. $N$ Engl J Med 370: 2071-2082. [Crossref]

11. Emura I, Usuda H (2016) Acute exacerbation of IPF has systemic consequences with multiple organ injury, with SRA ${ }^{+}$and TNF- $\alpha^{+}$cells in the systemic circulation playing central roles in multiple organ injury. BMC Pulm Med 16: 138. [Crossref]

12. Collard HR, Moore BB, Flaherty KR, Brown KK, Kaner RJ, et al. (2007) Acute exacerbations of idiopathic pulmonary fibrosis. Am J Respir Crit Care Med 176: 636643. [Crossref]

13. Raghu G, Collard HR, Egan JJ, Martinez FJ, Behr J, et al. (2011) An official ATS ERS/JRS/ALAT statement: idiopathic pulmonary fibrosis: evidence-based guidelines for diagnosis and management. Am J Respir Crit Care Med 183: 788-824.

14. Ambrosini V, Cancellieri A, Chilosi M, Zompatori M, Trisolini R, et al. (2003) Acute exacerbation of idiopathic pulmonary fibrosis: report of a series. Eur Respir J 22: 821826. [Crossref]

15. Rice AJ, Wells AU, Bouros D, du Bois RM, Hansell DM, et al. (2003) Terminal diffuse alveolar damage in relation to interstitial pneumonias. An autopsy study. Am J Clin Pathol 119: 709-714. [Crossref]

16. Emura I, Usuda H (2010) Histopathological and cytological examination of autopsy cases with multiple organ dysfunction syndromes. Pathol Int 60: 443-451. [Crossref]

17. Emura I, Usuda H (2011) Appearance of large scavenger receptor A positive cells and increased small scavenger receptor A positive cells in peripheral blood is associated with mortality in systemic inflammatory response syndrome and multiple organ dysfunction syndrome. Pathol Int 61: 7-12. [Crossref]

18. Naito M, Kodama T, Matsumoto A, Doi T, Takahashi K (1991) Tissue distribution, intracellular localization, and in vitro expression of Bovine macrophage scavenger receptor. Am J Pathol 139: 1411-1423. [Crossref]

19. Young DA, Lowe LD, Clark SC (1990) Comparison of the effects of IL-3, granulocytemacrophage colony-stimulating factor, and macrophage colony-stimulating factor in supporting monocytes differentiation in culture. J Immunol 145: 607-615. [Crossref]

20. Metcalf D (1988) The molecular control of blood cells. Harvard University Press, Cambridge. [Crossref]

21. Sakamoto K, Arakawa H, Mita S, Ishiko T, Ikei S, et al. (1994) Elevation of circulating interleukin 6 after surgery: factors influencing the serum level. Cytokine 6: 181-186. [Crossref]

22. Ware LB, Matthay MA (2000) The acute respiratory distress syndrome. $N$ Engl J Med 342: 1334-1349. [Crossref]

Copyright: (C2017 Emura I. This is an open-access article distributed under the terms of the Creative Commons Attribution License, which permits unrestricted use, distribution, and reproduction in any medium, provided the original author and source are credited. 